\title{
Effects of solar noise on the detection range performance of a laser spot tracker
}

\author{
Duck-Lae Kim $\oplus^{*} *$ Hyun-Bae Kong, and Seung-Tae Lee \\ LIG Nex1, EO/IR System R\&D, Yongin-si, Republic of Korea
}

\begin{abstract}
The effects of solar spectral irradiance on the performance of laser spot tracker are analyzed. When the entrance pupil diameter of the receiver increases, the field of view decreases, but the detection range increases. If the angle between the receiver and transmitter and target increases from 0 deg to $60 \mathrm{deg}$, the detection range decreases by approximately $23 \%$. Due to solar noise's effects caused by solar spectral irradiance, the detection range decreases by approximately $33 \%$ during the day, more than during the night, and the laser spot tracker's altitude decreases by approximately $13 \%$ at $2000 \mathrm{~m}$, more than at $0 \mathrm{~m}$. When the solar intensity's variation increases from $1 \%$ to $5 \%$, the detection range decreases by $54 \%$. (c) The Authors. Published by SPIE under a Creative Commons Attribution 4.0 Unported License. Distribution or reproduction of this work in whole or in part requires full attribution of the original publication, including its DOI. [DOI: 10.1117/1.OE.60.3.037102]
\end{abstract}

Keywords: optics; laser; optoelectronic system; solar noise; turbulence.

Paper 20210034 received Jan. 9, 2021; accepted for publication Feb. 24, 2021; published online Mar. 13, 2021.

\section{Introduction}

Laser light sources have been used in many fields such as medical, industrial, instrumentation, and defense along with increasing their output. Pulse-type lasers with high peak power are widely used for sensor measurement such as laser range finders, and continuous-type lasers with high average power are used for precision processing. ${ }^{1,2}$ The application areas of laser sensors vary according to the sensor types. Single-element detectors are used in laser range finders and array detectors are used for three-dimensional (3D) light detection and ranging. ${ }^{3,4}$ Quadrant detectors are used in devices that precisely measure laser beam coordinates such as laser spot trackers, laser autocollimators, high-speed reflector control systems, vibration and distortion monitoring devices, and atomic force microscopy used in semiconductor processes. Laser light sources used in laser spot trackers generally employ pulse lasers operating from several $\mathrm{Hz}$ to tens of $\mathrm{Hz}$, and laser receivers consist of a photodetector with an optical system, four photodiode arrays, and an amplifier that converts photocurrents into voltage and amplifies the voltage.$^{5-7}$ Although a constant signal can be acquired using a laser spot tracker at a fixed condition indoors, the laser signal received may vary according to the performance and position of the laser spot tracker and transmitter, target size, reflectance and angle, atmospheric conditions, and solar spectral irradiance using a laser spot tracker outdoors. In particular, solar spectral irradiance diminishes the detection range because it produces noise in addition to the hardware noise of the laser spot tracker. The fluctuation effect appears when light passes through a cloud and scatters, which has been measured as $0.6 \% .{ }^{8}$ The effects of the solar noise can be reduced using a bandpass filter's full width at half maximum (FWHM). To predict the performance of the detection range in a laser spot tracker in outdoor elements, it is necessary to analyze the solar noise.

In this study, the effects of characteristics of the optical system, angle between the laser spot tracker and transmitter, solar spectral irradiance, and solar intensity variations on the detection range of laser spot tracker were analyzed.

*Address all correspondence to Duck-Lae Kim, kimducklae@lignex1.com 


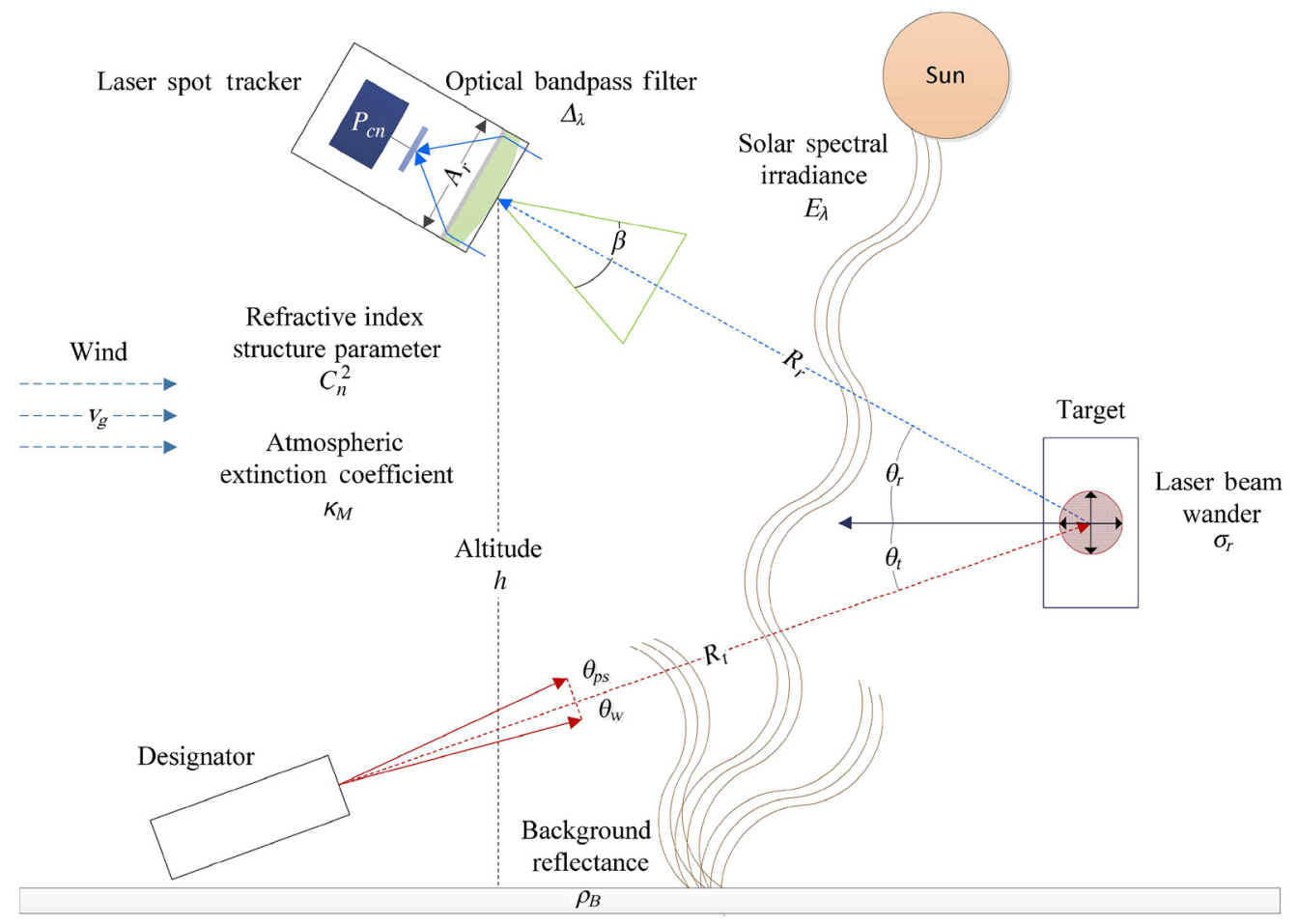

Fig. 1 Schematic diagram of model concept for laser spot tracker operation.

\section{Detection Range Model}

Figure 1 shows a schematic diagram of the model concept for the laser spot tracker operation. The laser designator, the receiver, the target, atmosphere, and position parameters for the detection range analysis of the laser spot tracker are shown in Fig. 1.

\subsection{Solar Spectral Irradiance}

The solar spectral irradiance measured at the ground increases from wavelengths of 350 to $500 \mathrm{~nm}$ to a maximum value of approximately $2250 \mathrm{Wm}^{-2} \mu \mathrm{m}^{-1}$ near $460 \mathrm{~nm}$ and then decreases above $500 \mathrm{~nm}$ to approximately $40 \mathrm{Wm}^{-2} \mu \mathrm{m}^{-1}$ at a $2400-\mathrm{nm}$ wavelength. ${ }^{9}$ The direct clear-sky irradiance regarding the altitude is expressed as follows: ${ }^{10}$

$$
E_{\lambda}(h)=I_{0}\left(1-A_{B}\left(h_{0}\right) \exp \left[-\alpha_{B}\left(h-h_{0}\right)\right]\right),
$$

where $h$ is the altitude, $I_{0}$ is the irradiance at sea level, $A_{B}\left(h_{0}\right)=1-\left(B_{c}\left(h_{0}\right) / I_{0}\right)$, and $\alpha_{B}=-\ln \left(\left[I_{0}-B_{c}\left(h_{H}\right)\right] /\left[I_{0}-B_{c}\left(h_{0}\right)\right]\right) /\left(h_{H}-h_{0}\right) . B_{c}\left(h_{0}\right)$ and $B_{c}\left(h_{H}\right)$ are the clear-sky irradiances at altitudes $h_{0}$ and $h_{H}$, respectively.

The solar spectral irradiance at a 1064-nm wavelength is $700 \mathrm{Wm}^{-2} \mu \mathrm{m}^{-1}$ at altitude $h_{0}$ and $900 \mathrm{Wm}^{-2} \mu \mathrm{m}^{-1}$ at altitude $h_{H}$. As the laser spot tracker's altitude increases, the solar spectral irradiance increases, thereby increasing the effects of the solar noise. When analyzing the detection range, the minimum and maximum altitudes of the laser spot tracker are 0 and $2000 \mathrm{~m}$, respectively.

\subsection{Atmospheric Extinction Coefficient}

When a laser beam propagates through the atmosphere, light extinction occurs due to atmospheric scattering. The atmospheric extinction coefficient varies according to the wavelength of the radiation and meteorological visual range, which is expressed by the following equation: ${ }^{11}$ 


$$
\kappa_{M}(\lambda) \approx \frac{3.91}{R_{v}}\left(\frac{550}{\lambda}\right)^{q}
$$

where $R_{v}$ is the visibility range and $\lambda$ is the wavelength. $q$ is calculated by $q=0.585 R_{v}^{1 / 3}$ when the visibility range is less than $6 \mathrm{~km}$ and 1.3 when the visibility range is the average seeing condition.

The visibility range varies depending on the aerosol distribution and is classified as 2 to $4 \mathrm{~km}$, 4 to $10 \mathrm{~km}$, and 10 to $20 \mathrm{~km}$ according to haze, light haze, and clear states. ${ }^{12}$ The extinction coefficient $\kappa_{M}$ is $0.166 \mathrm{~km}^{-1}$ when the visibility range is $10 \mathrm{~km}$, the laser wavelength is $1064 \mathrm{~nm}$, and the seeing conditions are average. This means that when a laser beam propagates through a $3-\mathrm{km}$ atmosphere, the laser light amount is attenuated exponentially to $39.2 \%$ attenuation. To analyze the detection range, $10 \mathrm{~km}$, which was the clear condition, was applied for the visibility range.

\subsection{Refractive Index Structural Parameter}

The refractive index structural parameter $C_{n}^{2}$ expresses the atmosphere's turbulence between the laser transmitter and target. The laser beam's divergence increases due to atmospheric turbulence as it propagates through the atmosphere. The Hufnagel-Valley, Miller-Zieske, and ITU-R models are used in the refractive index structural parameter model. $C_{n}^{2}$ tends to decrease as the altitude increases. $C_{n}^{2}$ regarding the altitude and wind velocity is expressed by the ITU-R model presented in the following equation: ${ }^{13}$

$$
C_{n}^{2}(h)=8.148 \times 10^{-56} v_{\mathrm{RMS}}^{2} h^{10} e^{-h / 1000}+2.7 \times 10^{-16} e^{-h / 1500}+C_{0} e^{-h / 100},
$$

where $C_{0}$ is the subaerial atmospheric structural constant, which has a typical value of $1.7 \times 10^{-14} \mathrm{~m}^{-2 / 3} . v_{\mathrm{RMS}}=\sqrt{v_{g}^{2}+30.69 v_{g}+348.91}$ is the root mean square wind velocity of the vertical path and $v_{g}$ is the subaerial wind velocity.

The wind velocity is classified as 5.5 to $7.9 \mathrm{~m} / \mathrm{s}, 8.0$ to $10.7 \mathrm{~m} / \mathrm{s}$, and 10.8 to $13.8 \mathrm{~m} / \mathrm{s}$ according to moderate breeze, fresh breeze, and strong breeze conditions. To analyze the detection range, $10 \mathrm{~m} / \mathrm{s}$, which was the fresh breeze condition, was applied for the subaerial wind velocity.

\subsection{Laser Beam Wander}

When a laser beam propagates through the atmosphere, if the turbulence cell is larger than the beam diameter, the laser beam's path is deflected and the beam's spot is displaced at the target, which is a certain distance away from the source. This is called laser beam wander, which is expressed by the following equation: ${ }^{14}$

$$
\sigma_{r}^{2}=1.83 C_{n}^{2} \lambda^{-1 / 6} R_{t}^{-17 / 6}
$$

where $C_{n}^{2}$ is the refractive index structural parameter, $\lambda$ is the laser beam's wavelength, and $R_{t}$ is the distance between the laser transmitter and target. The standard deviation in the $x$ or $y$ axis directions is expressed by $\sigma_{x}=\sigma_{y}=\left(\sigma_{r}^{2}\right)^{1 / 2}$.

To analyze the detection range, the standard deviation of the laser beam wander is set to $3 \sigma_{r}$ for a high laser energy density above $99 \%$. When a laser beam with a 1064-nm wavelength propagates through a 3000-m atmosphere in the horizontal direction at 1-m altitude, the standard deviation is $140.6 \mathrm{~nm}$.

\subsection{Background Optical Power}

When the laser spot tracker is used outdoors, the solar light reflecting from the target or ground surface is incident on the receiver's optical system. The background optical power received in the receiver acts as solar noise, affecting the detection range, which is expressed by the following equation: ${ }^{15}$ 


$$
P_{b}=\frac{\pi}{16} E_{\lambda} \Delta_{\lambda} \rho_{B} \beta^{2} A_{r}^{2} T_{r} T_{f} e^{-\kappa_{M} R_{r}} \cos \theta_{r},
$$

where $E_{\lambda}$ is the solar spectral irradiance, $\Delta_{\lambda}$ is the optical bandpass filter's bandwidth, $\rho_{B}$ is the background reflectance, $\beta$ is the receiver's field of view (FOV), $A_{r}$ is the receiver's entrance pupil diameter (EPD), $T_{r}$ is the transmission of the optical lens, $T_{f}$ is the optical bandpass filter's transmission, $\kappa_{M}$ is the atmospheric extinction coefficient, $R_{r}$ is the distance between the laser spot tracker and target, and $\theta_{r}$ is the angle between the normal target surface and the line joining the target and receiver centers.

The following values were applied to analyze the detection range: $60 \pm 10 \mathrm{~nm}$ for the bandwidth of optical bandpass filter, $50 \%$, which was the mean of reflectance $75 \%, 50 \%, 28 \%, 57 \%$, and $40 \%$ in a wall, building, road, soil, and branch for the background reflectance, ${ }^{16}$ approximately $40 \pm 10 \mathrm{deg}$ for the receiver's FOV, $27.5 \pm 7.5 \mathrm{~mm}$ for the receiver's EPD, $99.8 \%$ for the transmission of the optical lens, and $80 \%$ for the transmission of the optical bandpass filter.

\subsection{Diffuse and Pseudo-Specular Reflectance}

When a laser beam is vertically incident on the target, that is, the incident angle is $0 \mathrm{deg}$, it is reflected from the target similar to a Lambertian source. However, pseudo-specular reflectance occurs when the reflected beam is concentrated on the reflection angle area as the laser beam's incident angle increases. Pseudo-specular reflectance is clearly seen when the incident angle is above $60 \mathrm{deg}$ and the highest amount of light is reflected around the reflection angle when the incident angle is above $75 \mathrm{deg}$. The equation of the probability of diffuse reflection is expressed as follows: ${ }^{17}$

$$
\mathrm{P}_{\mathrm{D}}\left(\theta_{t}\right)=P_{0}-\frac{B}{W \sqrt{\frac{\pi}{2}}} \exp \left[-2 \frac{\left(\theta_{t}-\vartheta\right)^{2}}{W^{2}}\right]
$$

where $\theta_{t}$ is the angle between the laser transmitter and target, $P_{0}$ is the amount of normalized incident light, $B$ is $84.57, W$ is 52.81 , and $\vartheta$ is $103.20 \mathrm{deg}$.

$P_{D}\left(\theta_{t}\right)$ has the maximum value at an incident angle of $0 \mathrm{deg}$, which then decreases as the incident angle increases and becomes 0 because only pseudo-specular reflectance occurs at an incident angle above $85 \mathrm{deg}$. The probability of pseudo-specular reflection is expressed by $P_{P S}\left(\theta_{t}\right)=1-P_{D}\left(\theta_{t}\right)$.

\subsection{Signal Power}

When the laser beam is irradiated on the laser transmitter's target, the receiver in the laser spot tracker receives the laser signal power reflected from the target. The laser beam's divergence angle increases due to beam divergence, pointing stability, and turbulence. If the beam divergence angle increases, the laser beam's area that is incident on the target surface becomes larger than the target area, thereby generating spillover, which reduces the laser intensity reflected from the target and the laser spot tracker's receiver signals. When calculating the laser signal's power, Lambertian scattering, the target's cross-sectional area, target reflectance, and the angle between the target and receiver should be considered. The equation of the received optical signal's power is expressed as follows: ${ }^{12}$

$$
P_{r}=\left(\frac{P_{t}}{4 \pi R_{t}^{2}}\right)\left(e^{-\kappa_{M} R_{t}}\right)\left(\frac{1}{\theta_{d x}^{2}+\theta_{d y}^{2}+\theta_{p s}^{2}+\theta_{w}^{2}}\right) T_{t}\left(\frac{4 \rho_{T} A_{T} \cos \theta_{r}}{4 \pi R_{r}^{2}}\right)\left(e^{-\kappa_{M} R_{r}}\right) T_{r} T_{f} A_{r},
$$

where $P_{t}$ is the laser's peak power, $\theta_{d x}^{2}$ and $\theta_{d x}^{2}$ are the intrinsic laser beam's divergences in the $x$ and $y$ directions, respectively, $\theta_{p s}^{2}$ is the pointing stability, $\theta_{w}^{2}$ is the angle due to the laser beam wander, and $T_{t}$ is the transmittance of the laser transmitter's optics. $\rho_{T}$ is the target reflectance, $A_{T}$ is the target size, and $\theta_{r}$ is the angle between the target and laser spot tracker.

The following values were applied to analyze the detection range: approximately $3.9 \mathrm{MW}$, which divides the laser pulse energy of $70 \mathrm{~mJ}$ by the laser pulse width of $18 \mathrm{~ns}$ for the laser peak 
power $P_{t}, 3 \mathrm{~km}$ for $R_{t}$, which is the distance between the laser transmitter and target, $300 \mu \mathrm{rad}$ for both the beam divergence $\theta_{d x}^{2}$ and $\theta_{d x}^{2}, 100 \mu \mathrm{rad}$ for the pointing stability $\theta_{p s}^{2}, 100 \%$ for $T_{t}$, which is the transmittance of the laser transmitter's optics, and $10 \%$ for the target reflectance $\rho_{T}$.

\subsection{Signal-to-Noise Ratio}

The laser spot tracker receives not only the laser's signal power but also the background's optical power. Signal-to-noise ratio (SNR) is calculated using the received laser signals, shot noise due to the optical signals, shot noise due to the background radiation, noise due to the detector's dark currents, and other noise generated in the semiconductor. In this study, because the optical signal was a pulse-type signal, the shot noise due to the optical signal was ignored, and other noise generated in the semiconductor was also ignored, assuming that the value was negligibly small. The modulated background solar noise and circuit noise were added to the noise components to analyze the SNR's power ratio. The equation is expressed as follows: ${ }^{18}$

$$
\frac{S}{N}=\frac{\overline{I_{s g}^{2}}}{\overline{I_{\text {signal }}^{2}}+\overline{I_{\text {shot }}^{2}}+\overline{I_{\text {dark }}^{2}}+\overline{I_{\text {background }}^{2}}+\overline{I_{\text {circuit }}^{2}}},
$$

where $\overline{I_{s g}^{2}}=\frac{1}{2} R_{i}^{2} P_{r}^{2}$ is the laser signals, $R_{i}$ is the detector's responsivity, and $P_{r}$ is the optical system's received laser power. $\overline{I_{\text {signal }}^{2}}=2 e R_{i} P_{0} \Delta f_{m}$ is the shot noise due to the optical signal with an average current, $e$ is the electronic charge, $P_{0}$ is the optical signal's power, and $\Delta f_{m}$ is the signal's bandwidth. $\overline{I_{\text {shot }}^{2}}=2 e R_{i} P_{B} \Delta f_{m}$ is the shot noise due to background radiation. Because the background's optical power $P_{b}$ in Eq. (5) is incident on four channels in the detector in a distributed manner, it is expressed as $P_{B}=P_{b} / 2 . \overline{I_{\text {dark }}^{2}}=2 e I_{d k} \Delta f_{m}$ is the noise due to the detector's dark current, $I_{d k}^{2}=i_{d k 1}^{2}+i_{d k 2}^{2}+i_{d k 3}^{2}+i_{d k 4}^{2}$ is the total detector's dark current, and $i_{d k 4}$ in $i_{d k 1}$ is the dark current in every single channel at the quadrant detector.

$$
\overline{I_{\text {background }}^{2}}=\frac{1}{2} R_{i}^{2}\left(P_{B} P_{b f}\right)^{2}
$$

is the circuit noise, $P_{c n}^{2}=p_{c n 1}^{2}+p_{c n 2}^{2}+p_{c n 3}^{2}+p_{c n 4}^{2}$ is the total circuit noise's current, and $p_{c n 4}$ in $p_{c n 1}$ is the circuit noise in every single channel.

To analyze the detection range, because $R_{i} P_{0}$, which is the direct current (DC) component in $\overline{I_{\text {signal }}^{2}}=2 e R_{i} P_{0} \Delta f_{m}$ is eliminated, $\overline{I_{\text {signal }}^{2}}$ is ignored because the laser spot tracker acquires a pulsed laser signal. The following values were applied: $0.4 \mathrm{~A} / \mathrm{W}$ for the detector's responsivity $R_{i}, 1 \mathrm{MHz}$ for signal bandwidths $\Delta f_{m}, 200 \mathrm{nA}$ for the dark current $i_{d k}, 1 \%$ for the solar intensity variation $P_{b f}, 400 \mathrm{nW}$ for the circuit noise $p_{c n}$ at the single channel, and 10 for the SNR.

\section{Results}

Figure 2 shows the detection range and FOV for each bandpass filter according to the optical system's EPD. "ø" shows the six types of FOV according to the EPD, which was analyzed by CODE V, a commercial lens design tool. The dotted line is the FOV's fitting value. The analytical results demonstrated that as the EPD increased from 2.0 to $2.3,2.6,2.9,3.2$, and $3.5 \mathrm{~cm}$, the FOV decreased from $53 \mathrm{deg}$ to $47 \mathrm{deg}, 42 \mathrm{deg}, 38 \mathrm{deg}$, and $35 \mathrm{deg}$. The red, green, and blue lines indicate the FWHM's detection range at 50, 60, and $70 \mathrm{~nm}$ in each bandpass optical filter, respectively. The detection range calculated using Eqs. (1)-(8) tended to increase as the EPD increased. The EPD and FOV were fixed at $27.5 \mathrm{~mm}$ and $40 \mathrm{deg}$ to analyze the characteristics of the bandpass optical filter. When the transmittance of the bandpass optical filter was the same, the detection range increased as the FWHM decreased. This occurred because the solar noise decreased as the FWHM in the bandpass optical filter decreased. To analyze the detection range, the reference values were set as follows: $27.5 \mathrm{~mm}$ for the EPD of the receiver in the laser spot 


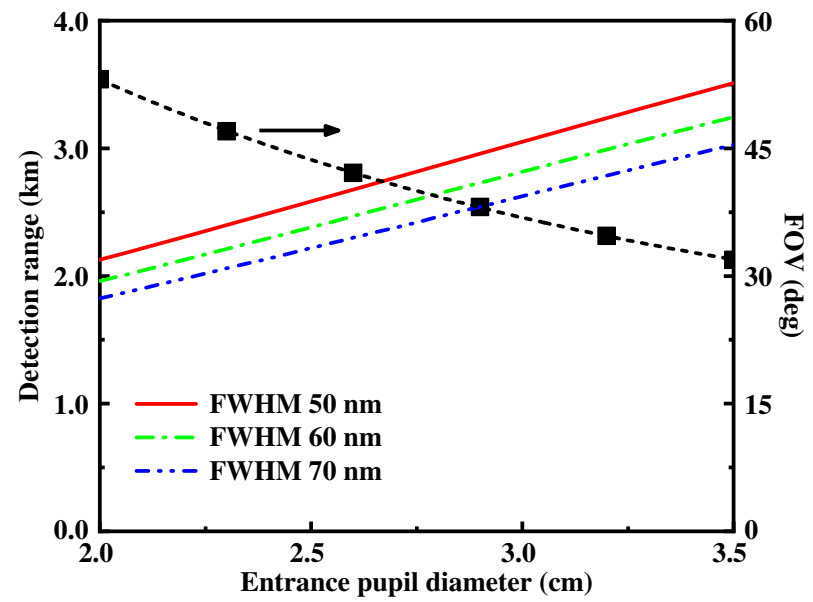

Fig. 2 The detection ranges and FOV as a function of the EPD according to the FWHM of the bandpass filter.

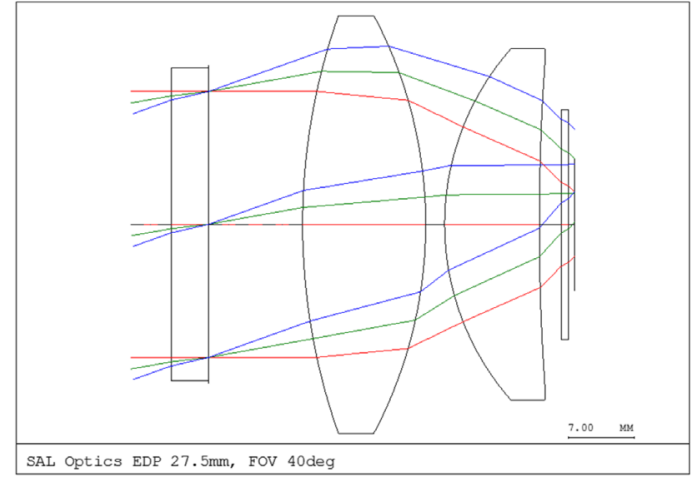

(a)

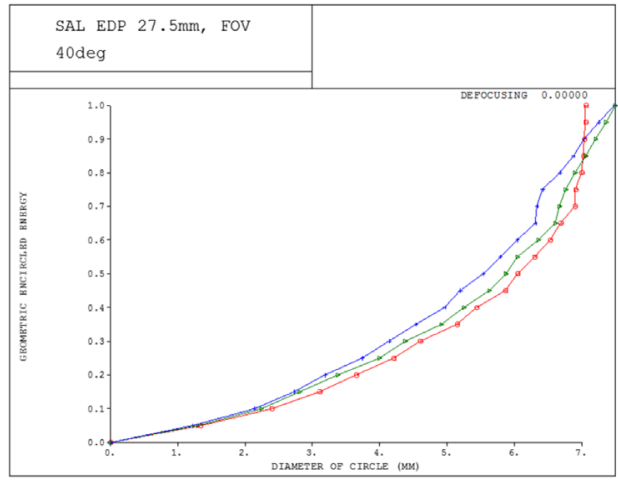

(b)

Fig. 3 Ray-tracing results of the designed optical system: (a) layout and (b) encircled energy.

tracker, 40 deg for the FOV, and $50 \mathrm{~nm}$ for the FWHM in the bandpass optical filter. The detailed lens design results are explained in Fig. 3.

Figure 3 shows the ray-tracing results in the optical system and encircled energy graph. Figure 3(a) shows the ray-tracing results in the optical system that satisfied the EPD's specification of $27.5 \mathrm{~mm}$ and FOV $40 \mathrm{deg}$, which were designed using CODE V. The optical system was composed of an optical window and two spherical lenses, and an aperture stop was arranged in the rear side of the optical window. The window in front of the image plane was a detecting window in the detector. The red line represents a bundle of rays whose incident angle was $0 \mathrm{deg}$, which was refracted by the lens, forming a spot-shaped image in the image plane. The green and blue lines represent the ray-tracing results whose incident angles were $10 \mathrm{deg}$ and $20 \mathrm{deg}$, respectively. From a $10 \mathrm{deg}$ or larger incident angle, the spot image that formed in the detection plane deviated from the detector range, which was characterized by energy loss. Figure 3(b) shows the geometric encircled energy graph, which verified that the spot formed for each FOV had an energy distribution according to the spot's diameter. The red, green, and blue lines show the energy distributions according to the spot's diameter at $0 \mathrm{deg}, 10 \mathrm{deg}$, and $20 \mathrm{deg}$ incident angles, respectively. Because the slope of the graph for each incident angle was similar, it was assumed that the change in the energy distribution according to the change in diameter would be similar.

Figure 4 shows the detection range according to the angle between the receiver (or transmitter) and target. The red line shows the detection range according to the angle between the receiver and target when fixing the angle between the transmitter and target to $0 \mathrm{deg}$. The green 


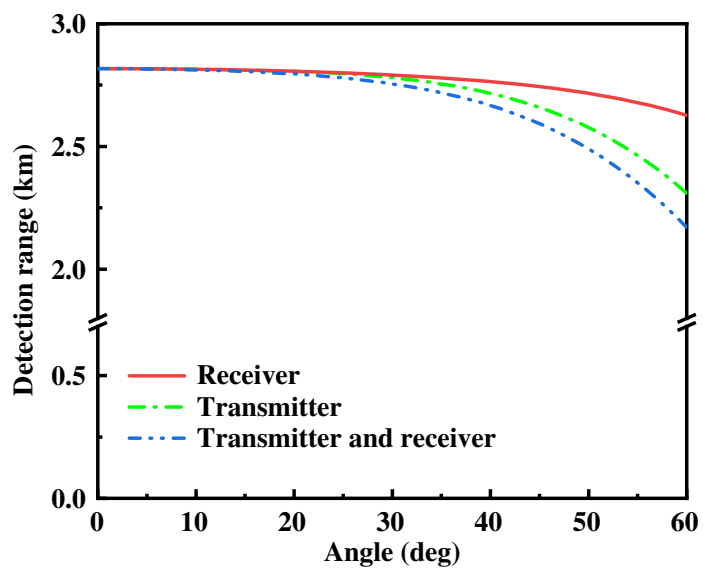

Fig. 4 The detection ranges as a function of the angle of the receiver and transmitter.

line shows the detection range according to the angle between the transmitter and target when fixing the angle between the receiver and target to $0 \mathrm{deg}$. The blue line shows the angle at which the receiver and transmitter changed in the same direction. When the angle of the receiver or transmitter was $0 \mathrm{deg}$, the detection range was $2817 \mathrm{~m}$, and the detection range tended to decrease as the angle of the receiver or transmitter increased. The calculated detection range was 2791 and $2627 \mathrm{~m}$ when the receiver angle was $30 \mathrm{deg}$ and $60 \mathrm{deg}, 2781$ and $2310 \mathrm{~m}$ when the transmitter angle was $30 \mathrm{deg}$ and $60 \mathrm{deg}$, and 2755 and $2170 \mathrm{~m}$ when the receiver and transmitter angles were $30 \mathrm{deg}$ and $60 \mathrm{deg}$, respectively. When the transmitter or receiver angle was $60 \mathrm{deg}$, the transmitter's detection range decreased 2.7 times more than the receiver. The detection range declined by approximately $1 \%, 3 \%$, and $5 \%$ when the transmitter's angles were approximately $28 \mathrm{deg}, 38 \mathrm{deg}$, and $44 \mathrm{deg}$ compared to $0 \mathrm{deg}$, and the detection range decreased by approximately $1 \%, 3 \%$, and $5 \%$ when the receiver's angles were approximately $31 \mathrm{deg}$, $47 \mathrm{deg}$, and $55 \mathrm{deg}$ compared to $0 \mathrm{deg}$ When the angle of the receiver and transmitter was $60 \mathrm{deg}$, the detection range declined by approximately $23 \%$ compared to that at the initial condition.

Figure 5 shows the detection range during the day and night according to the laser's output energy. The blue line shows the case during the day in the presence of the solar spectral irradiance effects, and the red line shows the case at night without the solar noise's effects. The detection range was 2390, 2817, and $3183 \mathrm{~m}$ during the day and 3764, 4271, and $4681 \mathrm{~m}$ at night when the laser's output energy increased from 50 to 70 and $90 \mathrm{~mJ}$, respectively. The detection range decreased by approximately $33 \%$ due to the background solar noise's effects during the day. The detection range decreased as the solar spectral irradiance increased.

Figure 6 shows the detection range and solar spectral irradiance according to the receiver's altitude. The red line shows the solar spectral irradiance according to the altitude, which was

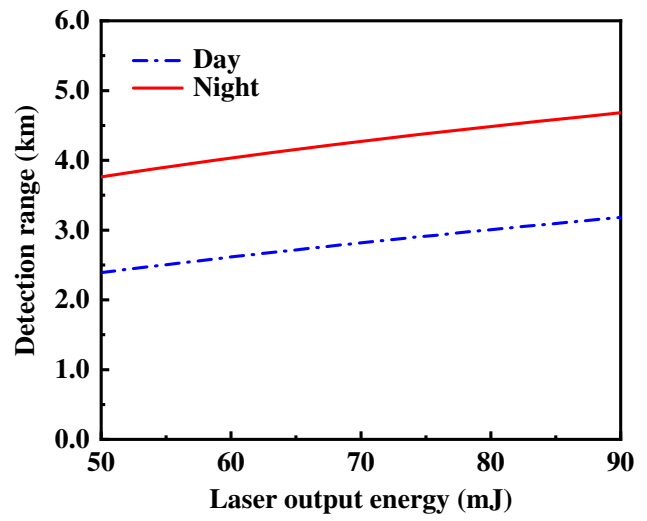

Fig. 5 The detection ranges as a function of the laser's output energy according to the day or night. 


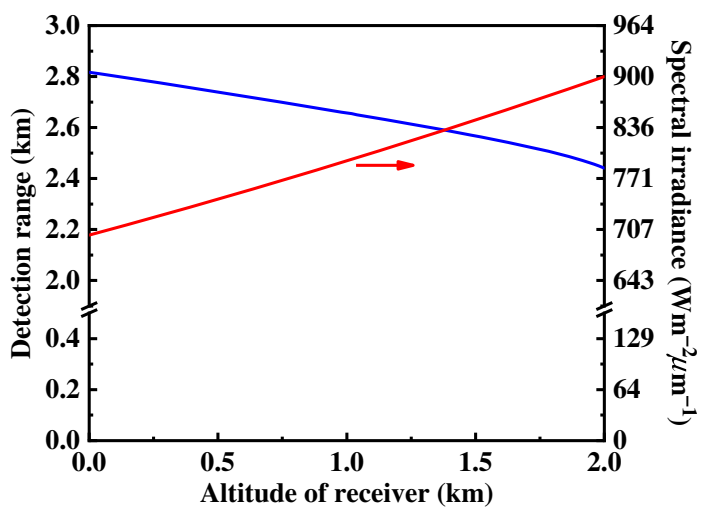

Fig. 6 The detection range and solar spectral irradiance as a function of the receiver's altitude.

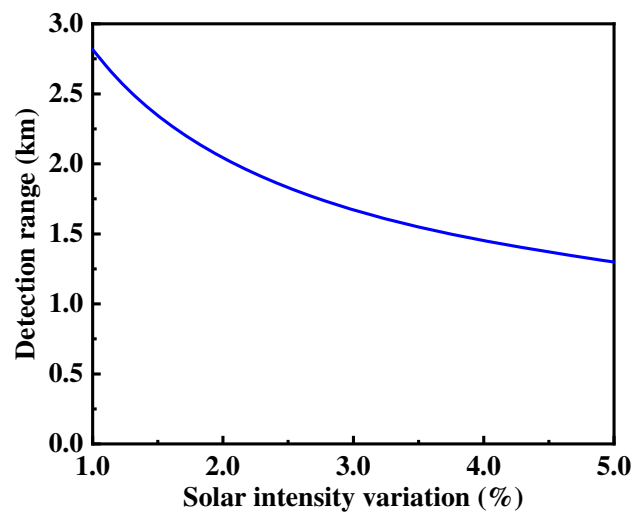

Fig. 7 The detection range as a function of the solar intensity's variation.

calculated using Eq. (1). The blue line shows the detection range of the solar spectral irradiance according to the altitude. The solar spectral irradiance tended to increase, which was $700 \mathrm{Wm}^{-2} \mu \mathrm{m}^{-1}$ at the ground and $900 \mathrm{Wm}^{-2} \mu \mathrm{m}^{-1}$ at an altitude of $2000 \mathrm{~m}$. This occurred because the atmospheric effect was less influential as the altitude increased, increasing the solar spectral irradiance. The detection range decreased by approximately $13 \%$ due to the solar noise's effects at an altitude of $2000 \mathrm{~m}$ compared to that at the sea level. Because there was no effect of solar spectral irradiance at night, there was no change in the detection range due to the altitude.

Figure 7 shows the detection range according to the solar intensity's variation. The solar intensity's variation is $P_{b f}$ in the $\overline{I_{\text {background }}^{2}}$ term in Eq. (8). When the solar intensity's variation increased from $1 \%$ to $5 \%$, the detection range decreased by 54\% from 2817 to $1298 \mathrm{~m}$. In the outdoors, the detection range of the laser spot tracker decreased because of the increase in the solar noise as the solar spectral irradiance increased. The detection range also decreased even when the solar intensity variation increased because of the increase in the receiver noise. The solar noise was reduced by decreasing the FWHM of the bandpass optical filter, but the laser spot tracker's performance was optimized when the reduction in the transmittance according to the reduction in FWHM of the bandpass optical filter was considered together.

\section{Conclusion}

In this study, the effects of EPD and FOV in an optical system, the angles between the receiver and transmitter, the solar spectral irradiance, and the effects of solar intensity's variation on the detection range of a laser spot tracker were analyzed. When the EPD increased from 2 to $3.5 \mathrm{~cm}$, the FOV decreased from $53 \mathrm{deg}$ to $35 \mathrm{deg}$, and the detection range increased as the FWHM in the 
bandpass optical filter was reduced from 70 to $50 \mathrm{~nm}$. The EPD and FOV of the receiver in the laser spot tracker were set to $27.5 \mathrm{~mm}$ and $40 \mathrm{deg}$ and the FWHM in the bandpass optical filter was set to $50 \mathrm{~nm}$ to analyze the detection range. As the angles of the receiver and transmitter increased, the detection range decreased by approximately $23 \%$ at $60 \mathrm{deg}$ compared to at $0 \mathrm{deg}$. The detection range during the day declined by approximately $33 \%$ compared to that at night due to the solar noise's effects. As the altitude of the laser spot tracker increased, the solar spectral irradiance increased, and the detection range decreased by approximately $13 \%$ at an altitude of $2000 \mathrm{~m}$ compared to that at an altitude of $0 \mathrm{~m}$. The analysis results showed that when the solar intensity's variation increased from $1 \%$ to $5 \%$, the detection range decreased by $54 \%$. In future studies, experimental analyses should be based on the theoretical results of the reduction in the detection range due to solar noise.

\section{References}

1. W. Koechner, Solid-State Laser Engineering, Springer, New York (2006).

2. K. J. Kuhn, Laser Engineering, Prentice-Hall, Upper Saddle River, New Jersey (1998).

3. S. Kruapech and J. Widjaja, "Laser range finder using Gaussian beam range equation," Opt. Laser Technol. 42, 749-754 (2010).

4. D.-L. Kim et al., "Common optical system for the fusion of three-dimensional images and infrared images," Curr. Opt. Photonics 3(1), 8-15 (2019).

5. Z. P. Barbaric et al., "New relationship of displacement signal at quadrant photodiode: control signal analysis and simulation of a laser tracker," Optik 125, 1550-1557 (2014).

6. Y. Juqing, W. Dayonga, and Z. Weihu, "Precision laser tracking servo control system for moving target position measurement," Optik 131, 994-1002 (2017).

7. Q. Vo, X. Zhang, and F. Fang, "Extended the linear measurement range of four-quadrant detector by using modified polynomial fitting algorithm in micro-displacement measuring system," Opt. Laser Technol. 112, 332-338 (2016).

8. N. Silsirivanich, "Fluctuation characteristics effect analysis of solar irradiance data by wavelet transform," Energy Procedia 138, 301-306 (2017).

9. L. Zhang et al., "The effects of solar irradiance spectra on calculation of narrow band top-ofatmosphere reflectance," IEEE J. Sel. Top. Appl. Earth Obs. Remote Sens. 7(1), 49-58 (2014).

10. A. Oumbe et al., "Solar irradiance in clear atmosphere: study of parameterisations of change with altitude," Adv. Sci. Res. 6, 199-203 (2011).

11. R. M. Measures, Laser Remote Sensing Fundamentals and Applications, Krieger Publishing Company, Malabar, Florida (1992).

12. G. C. Holst, Holst's Practical Guide to Electro-Optical Systems, JCD Publishing Company, Winter Park, Florida (2003).

13. H.-Y. Wei and Z.-S. Wu, "Study on the effect of laser beam propagation on the slant path through atmospheric turbulence," J. Electromagn. Waves Appl. 22, 787-802 (2008).

14. H. Weichel, Laser Beam Propagation in the Atmosphere, SPIE Press, Bellingham, Washington (1990).

15. L. M. Manojlovic and Ž. P. Barbaric, "Optimization of optical receiver parameters for pulsed laser-tracking systems,” IEEE Trans. Instrum. Meas. 58(3), 681-690 (2009).

16. K. Tan, X. Cheng, and X. Cheng, "Modeling hemispherical reflectance for natural surfaces based on terrestrial laser scanning backscattered intensity data," Opt. Express 24(20), 22971-22988 (2016).

17. A. Earp, G. Smith, and J. Franklin, "Simplified BRDF of a non-Lambertian diffuse surface," Lighting Res. Technol. 39(3), 265-281 (2007).

18. C.-L. Chen, Elements of Optoelectronics and Fiber Optics, IRWIN, Chicago (1996).

Duck-Lae Kim is a research engineer at LIG Nex1. He received his BS and MS degrees in physical optics from Cheongju University in 2002 and 2004, respectively, and his PhD in applied optics from Dankook University in 2008. He is the author of more than 15 journal papers. His current research interests include high-power lasers and optoelectronic systems.

Biographies of the other authors are not available. 